\title{
Mutants of the Zinc Ligands of Lacticin 481 Synthetase Retain Dehydration Activity but Have Impaired Cyclization Activity
}

\author{
Supporting Information
}

Moushumi Paul, Gregory C. Patton, and Wilfred A. van der Donk ${ }^{*}$

\section{MAterials ANd Methods}

\section{General}

UV-vis spectra were obtained using a Hewlett Packard model 8453 UV-vis spectrophotometer or a Cary 100 Bio UV-visible spectrophotometer (Varian). Centrifugation was performed using either a bench-top Beckman Centrifuge model GS6R with a GA-10 rotor, a Beckman Coulter Centrifuge model J2-HS with a JA-20 or JLA 10.5 rotor, or an Eppendorf minifuge. Sonication was carried out using a Sonics and Materials, Inc. Vibra Cell model sonicator. Protein purification used peristaltic pumps, model P-1, and RediFrac fraction collectors produced by Pharmacia Biotech or a BioCAD fast protein liquid chromatography (FPLC) system.

Matrix assisted laser desorption ionization - time of flight (MALDI-TOF) mass spectrometry was carried out in the Mass Spectrometry Laboratory at the University of Illinois, using a Voyager-DE STR mass spectrometer. For samples with no salt (i.e. HPLC purified samples), sinapinic acid was utilized as the matrix, while salt-containing samples used $\alpha$-cyano-4-hydroxy-cinnamic acid as matrix.

Reverse phase - high performance liquid chromatography (RP-HPLC) was carried out using a Rainin system (Dynamax model SD-200 pump and model UV-1 detector) or a Beckman Gold system (Model 125 solvent module and model 166 detector) using a Vydac C-4 or C-18 analytical column $(25 \mathrm{~cm} \mathrm{x} 0.46 \mathrm{~cm})$, monitoring absorbance at 220 nm. Preparative RP-HPLC was carried out using a Waters system (Model 600 controller, Delta 600 pump and 2487 dual wavelength absorbance detector) using a Waters PrepLC ${ }^{\text {TM }} 25 \mathrm{~mm}$ Module C-4 or C-18 preparative column, monitoring absorbance at 220 $\mathrm{nm}$. Solvent A consisted of $0.1 \%$ trifluoroacetic acid (TFA) in water and the components of solvent B were $80 \%$ acetonitrile, $20 \%$ water and $0.086 \%$ TFA.

\section{Oligonucleotide primer sequences for site directed mutagenesis}

Primers used for generation of H725N, C781A, C836A, and C781A/C836A LctM. Mutations are underlined.

Cys781rev:GTCCCGATGCACCGTGAGCCCATTGCGAAGAGTATGAAC Cys781for:GTTCATACTCTTCGCAATGGGCTCACGGTGCATCGGGAC Cys836for:GTATACAGATAATTTTTGTCTAGCTCATGGTATTTTAGG Cys836rev:CCTAAAATACCATGAGCTAGACAAAAATTATCTGTATAC H725N-for: GTATTGCAAGTTATGCTAATGGTAATAGTGG H725N-for: CCACTATTACCATTAGCATAACTTG CAATAC 


\section{Mass spectrometry data}

His $_{6}$-LctA treated with PHMB. MALDI-TOF MS calcd. 7709 (M), 7909 (M+200(Hg)), $8029(\mathrm{M}+320(\mathrm{HgAr})), 8229(\mathrm{M}+200+320), 8349(\mathrm{M}+640), 8669(\mathrm{M}+960)$, found 7908 $(\mathrm{M}+200), 8030(\mathrm{M}+320), 8229(\mathrm{M}+200+320), 8352(\mathrm{M}+640), 8679(\mathrm{M}+960)$. See figure S1.

His $_{6}$-LctA(1-38)S35A reacted with PHMB. MALDI-TOF MS calcd. 6145 (M), 6465 $(\mathrm{M}+320)$; found $6145(\mathrm{M}), 6466(\mathrm{M}+320)$.

His $_{6}$-LctA(1-38)S35A reacted with wt LctM. MALDI-TOF MS calcd. 6145 (M), 6127 (M-18), found 6124 (M-18).

His $_{6}$-LctA(1-38)S35A reacted with LctM H725N. MALDI-TOF MS calcd. 6145 (M), 6127 (M-18), found 6128 (M-18).

His $_{6}$-LctA(1-38)S35A reacted with LctM C781A. MALDI-TOF MS calcd. 6145 (M), 6127 (M-18), found 6127 (M-18).

His $_{6}$-LctA(1-38)S35A reacted with LctM C836A. MALDI-TOF MS calcd. 6145 (M), 6127 (M-18), found 6128 (M-18).

His $_{6}$-LctA(1-38)S35A reacted with LctM C781A/C836A. MALDI-TOF MS calcd. 6145 (M), 6127 (M-18), found 6127 (M-18).

His $_{6}-$ LctA(1-38)S35A reacted with wt LctM and treated with PHMB. MALDI-TOF MS calcd. 6145 (M), 6127 (M-18), 6447 (M-18+320), found 6126 (M-18).

His $_{6}$-LctA(1-38)S35A reacted with LctM H725N and treated with PHMB. MALDITOF MS calcd. 6145 (M), 6127 (M-18), 6447 (M-18+320), found 6128 (M-18), 6450 (M-18+320).

His $_{6}$-LctA(1-38)S35A reacted with LctM C781A and treated with PHMB. MALDITOF MS calcd. 6145 (M), 6127 (M-18), 6447 (M-18+320), found 6127 (M-18), 6447 (M-18+320).

His $_{6}$-LctA(1-38)S35A reacted with LctM C836A and treated with PHMB. MALDITOF MS calcd. 6145 (M), 6127 (M-18), 6447 (M-18+320), found 6127 (M-18), 6447 (M-18+320).

His $_{6}$-LctA(1-38)S35A reacted with LctM C781A/C836A and treated with PHMB. MALDI-TOF MS calcd. 6145 (M), 6127 (M-18), 6447 (M-18+320), found 6126 (M-18), $6446(\mathrm{M}-18+320)$. 
Figure S1. Treatment of unmodified LctA with PHMB yields an array of arylmercury and mercury adducts. A single mercury adduct corresponds to a bridging mercury atom between two cysteine residues $(\mathrm{M}+\mathrm{Hg})$; mercury aryl adducts are also formed at one $(\mathrm{M}$ $+\mathrm{HgAr})$, two $(\mathrm{M}+2 \mathrm{HgAr})$ and three $(\mathrm{M}+3 \mathrm{HgAr})$ cysteines in the substrate; finally a bridging mercury atom between two cysteines and a single mercury aryl adduct $(\mathrm{M}+\mathrm{Hg}$ $+\mathrm{HgAr}$ ) is also observed at 8,229 Da.

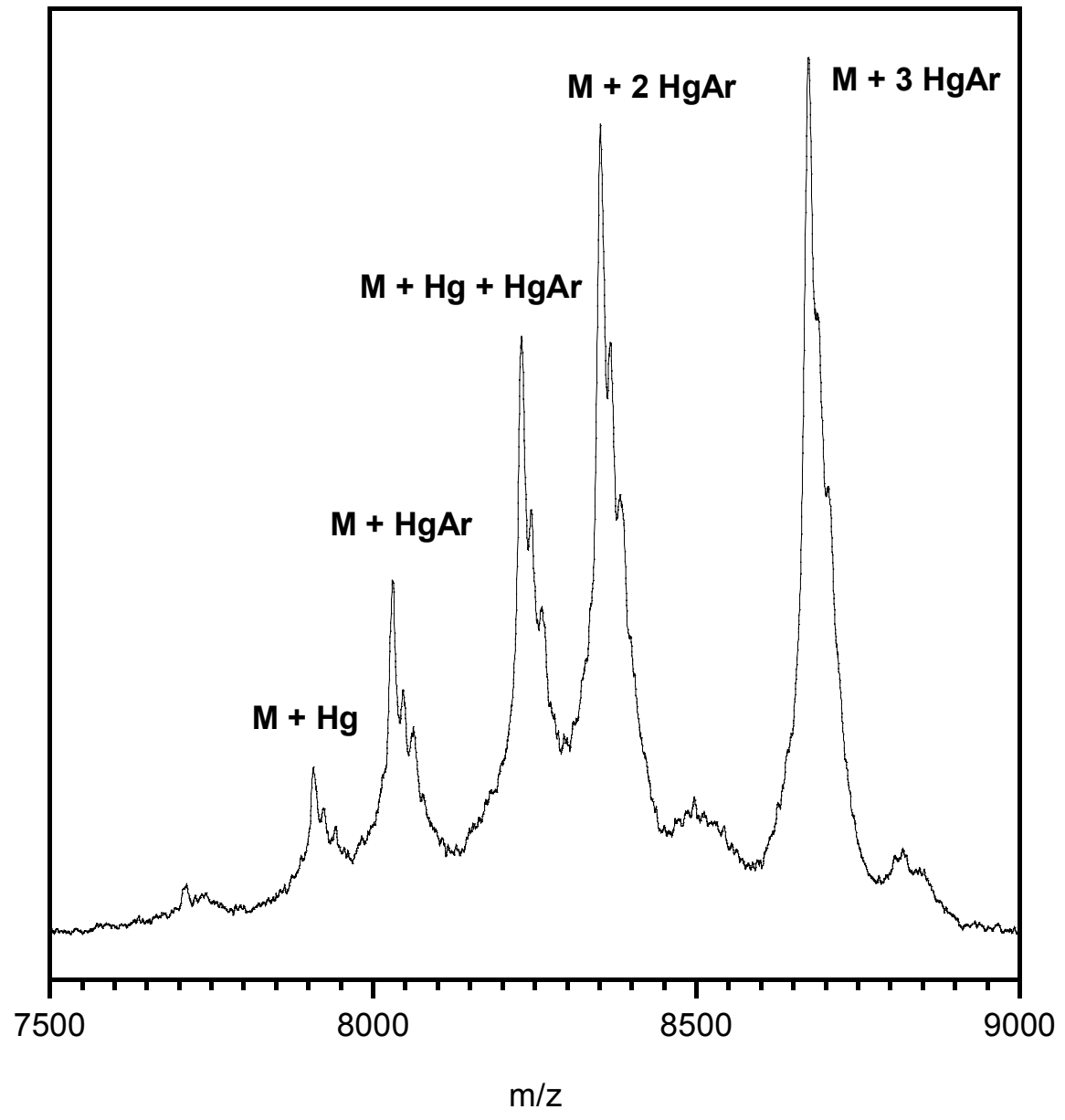


Figure S2. MALDI-TOF MS of LctA(1-38)S35A before (solid) and after treatment with PHMB (dashed). Near quantitative modification of cysteine 38 by PHMB is observed for the unprocessed substrate.

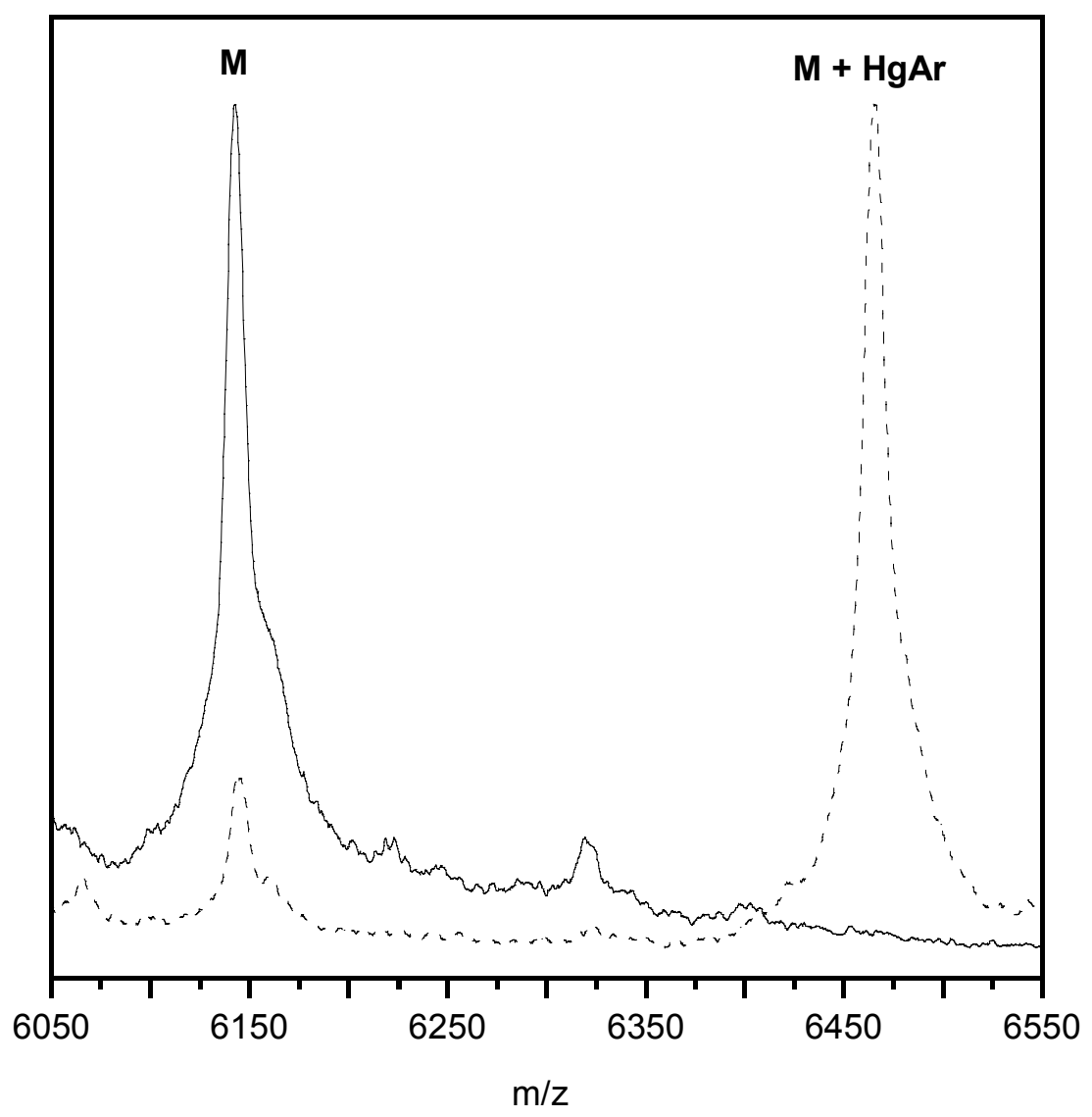

